\title{
Stereotactic body radiotherapy (SBRT) for central and ultracentral node-negative lung tumors
}

\author{
Dawn Owen ${ }^{1}$, Terence T. Sio ${ }^{2}$ \\ ${ }^{1}$ Department of Radiation Oncology, Mayo Clinic, Rochester, Minnesota, USA; ${ }^{2}$ Department of Radiation Oncology, Mayo Clinic, Phoenix, \\ Arizona, USA \\ Contributions: (I) Conception and design: All authors; (II) Administrative support: None; (III) Provision of study materials or patients: All authors; \\ (IV) Collection and assembly of data: All authors; (V) Data analysis and interpretation: All authors; (VI) Manuscript writing: All authors; (VII) Final \\ approval of manuscript: All authors. \\ Correspondence to: Dawn Owen, MD, PhD. Department of Radiation Oncology, Mayo Clinic, 200 1st St SW, Rochester, MN 55905, USA. \\ Email: Owen.Dawn@mayo.edu.
}

\begin{abstract}
Advancements in imaging and radiotherapy (RT) techniques have allowed for remarkably precise delivery of high radiation dose per treatment fraction to intrathoracic targets. As a non-invasive therapeutic modality (compared to surgery), stereotactic body radiotherapy (SBRT) is an attractive option for patients with early-stage non-small cell lung cancers and oligometastases, especially for older patients with significant comorbidities and pre-existing pulmonary dysfunction. However, the outcomes and side effect profile of SBRT are highly dependent on tumor location, especially if the tumor is located centrally (within $2 \mathrm{~cm}$ of the proximal bronchial tree (PBT)] or ultracentrally (touching or within $1 \mathrm{~cm}$ of the mediastinum, esophagus, and PBT). In this focused review, we will examine the contemporary practice and principles of using hypofractionated RT or SBRT for central and ultracentral thoracic tumors. We will identify future directions on how this practice may be incorporated into the increasingly complicated modern paradigm of lung cancer treatments which now include immunotherapy along with proton beam radiotherapy.
\end{abstract}

Keywords: Stereotactic body radiotherapy (SBRT); SABR; lung cancer; central; ultracentral; local control; toxicities; review

Submitted Mar 16, 2020. Accepted for publication Apr 20, 2020.

doi: $10.21037 /$ jtd-2019-cptn-01

View this article at: http://dx.doi.org/10.21037/jtd-2019-cptn-01

\section{Introduction}

In the modern era, stereotactic body radiotherapy (SBRT) is a powerful tool that can deliver ablative, tumoricidal radiation dose over a convenient schedule of typically 3 to 5 treatments. SBRT is commonly applied for thoracic malignancies, and is the standard of care for patients with early-stage, inoperable non-small cell lung cancer $(1,2)$. The delivery of SBRT requires significant input from medical physics and dosimetry: it necessitates accurate tumoral and organ structure mapping with tight margins (stereotaxy), sophisticated intensity modulated radiotherapy techniques to spare normal organs and to target the tumor, the ability to deliver a higher dose rate of radiation by a linear accelerator, and also sophisticated imaging (image-guided radiotherapy, IGRT) and respiratory motion management techniques (3).

For thoracic targets, including primary lung cancers and metastases, the anticipated side effects (or toxicities) of SBRT are most dependent on the location of the tumor, namely peripheral versus central location (4) (Figure 1). In the radiation oncology literature, we are now separating lesions into central and ultracentral locations, as an ultracentral location may confer a higher risk of toxicity. In this focused review, we will examine the current understanding and clinical pearls of management of central and ultracentral thoracic tumors. We will also point out the existing challenges and controversies while highlighting 


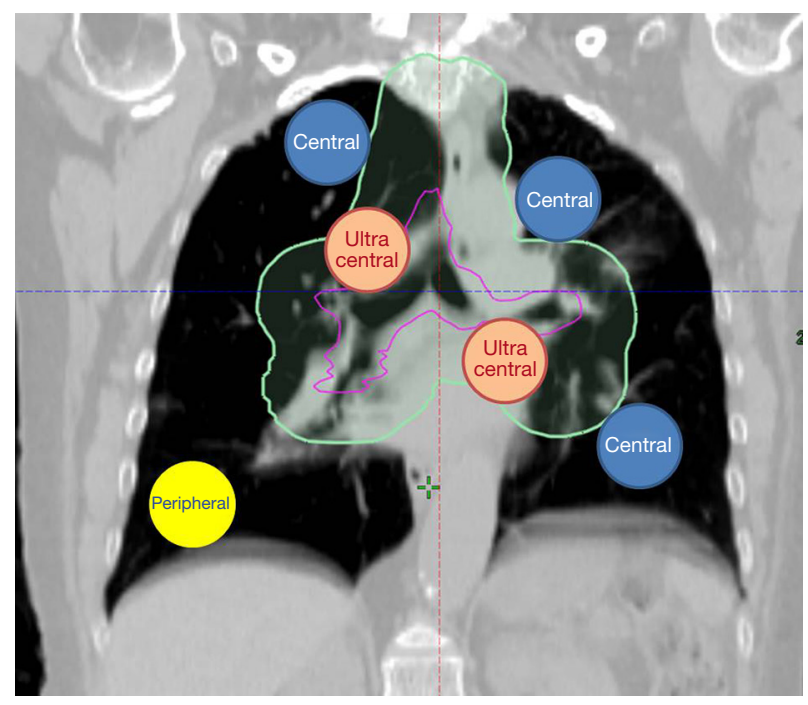

Figure 1 Inner outline (pink) is contour of proximal bronchial tree (PBT). Outer line (light green) is a 2 -cm expansion on PBT. Central lesions are defined as those which abut this $2 \mathrm{~cm}$ expansion and touching heart and great vessels. Ultracentral lesions lie within $1 \mathrm{~cm}$ of the PBT and include lesions that abut esophagus and trachea. In this illustration, the ultracentral lesions about the proximal bronchial tree and lie within the expansion of the PBT and the $2 \mathrm{~cm}$ expansion outlined in light green. Peripheral lesions lie well outside of the outer line (light green).

potential improvements in radiation techniques (such as proton beam radiotherapy) and their relation to systemic therapy advances including immunotherapy for lung cancer.

\section{Definition of central and ultracentral tumors}

The radiation oncology definition of a central tumor is a tumor that lies within or is touching the zone of a uniform, $2-\mathrm{cm}$ expansion around the proximal bronchial tree (PBT) or immediately adjacent to the pleura covering the mediastinum or pericardium $(4,5)$ (Figures 1,2). The nuance of an ultracentral tumor is that the margin is narrowed to a $1 \mathrm{~cm}$ expansion of the PBT, and the lesion immediately touches or invades one of the organs at-risk such as the mediastinum, trachea, bronchus, or esophagus (6) (Figure 3). In practice, the $\mathrm{PBT}$ structure is contoured on axial CT slices averaged from a 10-phase 4D-CT scan, and it includes the distal $2 \mathrm{~cm}$ of the trachea, the carina, the bilateral (left and right) mainstem bronchi, the bilateral upper lobe bronchi, the bronchus intermedius leading to the right middle lobe bronchus, the bilateral lower lobe bronchi, and

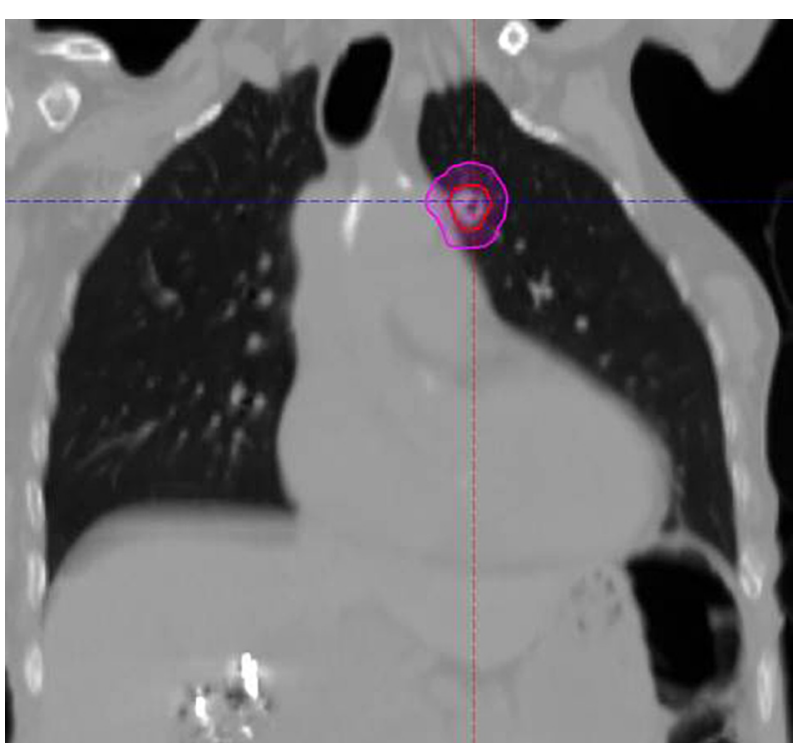

Figure 2 Example of a central lesion treated with SBRT. This lesion is not within the $2 \mathrm{~cm}$ expansion of the proximal bronchial tree. However, it abuts the aorta. Central lesions also include those that abut the heart and great vessels.

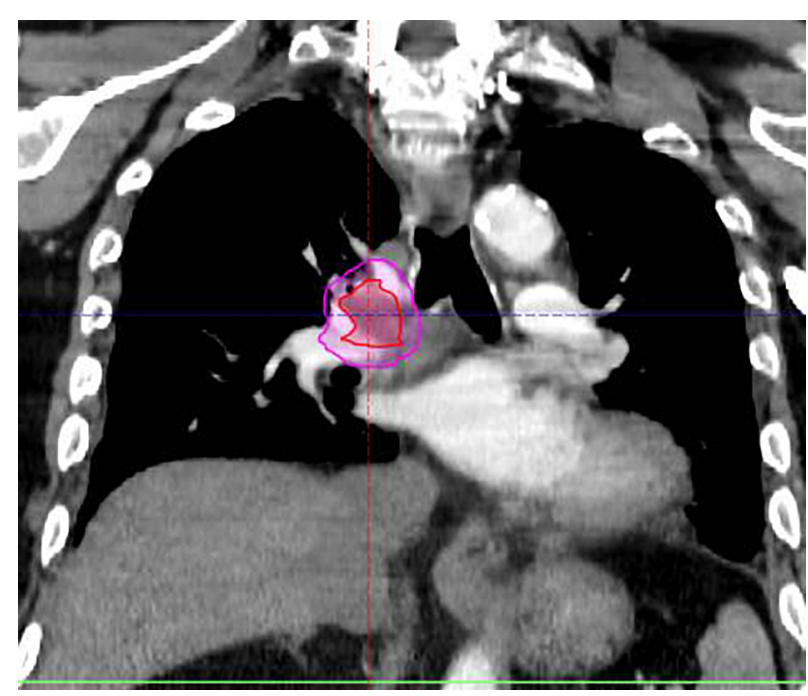

Figure 3 Example of an ultracentral lesion treated with SBRT. This lesion is located adjacent to bronchus and esophagus. Red is the gross tumor volume. Pink is the planning target volume (the volume to which we prescribe our dose).

the lingular bronchus (a left lung structure). This structure is then expanded isometrically by 1 and $2 \mathrm{~cm}$, to be visually compared and verified with the tumor volumes that are drawn by the treating radiation oncologist. It is important 


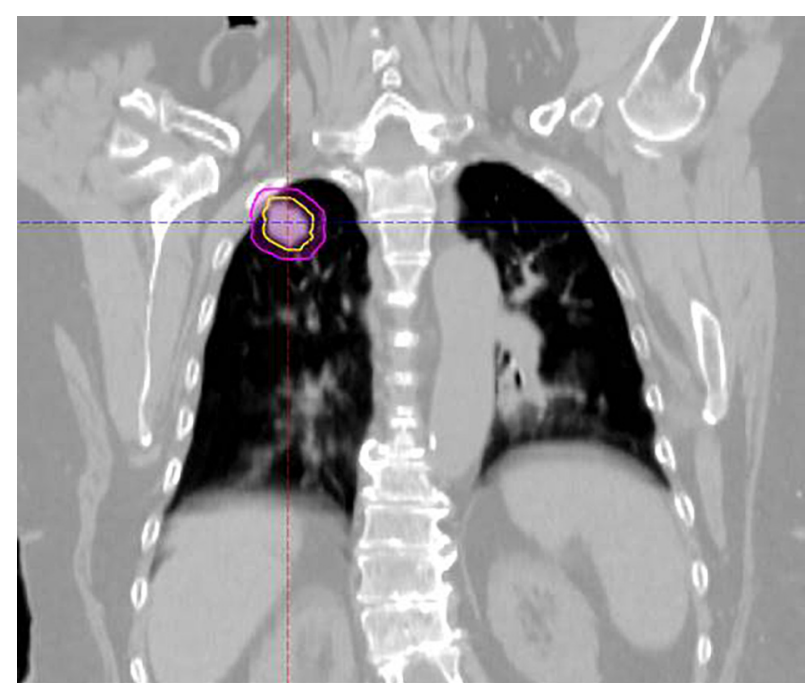

Figure 4 Example of a peripheral lesion treated with SBRT. This lesion is not close to any vessels, heart or within $2 \mathrm{~cm}$ of the proximal bronchial tree.

to note that, any part of the tumoral volume overlapping or touching this expanded PBT structure (or mediastinum) would qualify as a central or ultracentral tumor, according to the definitions noted above. This is of particular importance during SBRT dosimetric planning, as it impacts radiation dosing. For example, a peripheral tumor (i.e., not central or ultracentral) can be treated with much higher doses per fraction and over a shorter radiation course, e.g., 54 Gray (Gy) in 3 fractions or 48 Gy in 4 fractions $(2,7)$ (Figure 4). Additional details on dose and fractionation for central and ultracentral lesions are discussed below.

\section{Workup and treatment options}

As with any primary lung neoplasm, staging is vital for determining the paradigm of treatment. By definition, central and ultracentral lesions would likely be T2NX and above disease given that $\mathrm{T} 2$ disease already encompasses lesions that invade the main bronchus and extend to the hilum $(8,9)$.

As per the National Comprehensive Cancer Network (NCCN) guidelines, one would begin with computerized tomography (CT) chest with and without IV contrast, biopsy, positron emission tomography-computed tomography (PET-CT), surgical mediastinal staging (mediastinoscopy or endobronchial ultrasound/EBUS), and pulmonary function tests. Mediastinal node staging is critical as there can be occult metastases to N1 and N2 nodes in central and ultracentral lesions. Endobronchial ultrasound with biopsy of suspicious/enlarged nodes should be strongly considered (10-12). Brain magnetic resonance imaging (MRI) or head CT with contrast should be considered as well; especially for stage II and above nonsmall cell histologies, all histologically confirmed small cell lung cancer $(13,14)$ or any patient who may be symptomatic.

The nuance of central and ultracentral lesions lies in determining whether definitive surgery or radiotherapy/ chemoradiation are potential options. The standard of care for resection is lobectomy of the involved lobe and mediastinal node dissection/sampling. The decision for resection must occur in a multidisciplinary setting; particularly with ultracentral lesions, as surgery may require a pneumonectomy which the patient may not tolerate depending on performance status and pulmonary function at diagnosis, or the patient may refuse.

For lesions whose radiation volume will overlap esophagus, main bronchus, great vessels, or trachea, part of the workup includes ruling out any direct invasion of these organs. A CT angiogram or MR angiogram can be very useful for identifying direct vascular invasion. Direct visualization of the trachea and bronchial tree via endoscopy is also recommended to identify any endoluminal studding which can affect both the delineation of the gross tumor volume as well as the clinical target volume. Upper endoscopy can also determine if there is any direct invasion of the esophagus. For cases where the pericardium-tumor interface is compromised, MR of the heart can be very informative for early cardiac muscle invasion. Knowing the extent of disease in advance informs risk, especially if one is considering stereotactic radiation as the risk of fistula or fatal hemorrhage would be very high in the scenario where there is direct tumor invasion (15-18).

There is an increasing body of literature now examining late cardiac effects of radiation and this is especially pertinent to central and ultracentral lesions where the heart dose is particularly high (19-24). Most of the literature comes from recent publications on conventionally delivered radiation in locally advanced lung cancer in which excess mean heart dose (>11-20 Gy) resulted in higher grade 3 cardiac events defined as: acute coronary syndrome, new congestive heart failure, cardiac arrest, new onset arrhythmia, valvular disease, and new pericardial effusion (19-24). In the randomized dose escalation radiation trial for locally advanced lung cancer, RTOG 0617, there was worse overall survival in the dose escalation group that was attributable to higher mean heart dose from radiotherapy 
$(25,26)$. In the era of immunotherapy where survival from Stage III lung cancer has doubled and in patients who are receiving SBRT for localized node-negative disease, it may be a consideration to optimize cardiac status prior to and after delivery of radiation. The long-term cardiac effects of SBRT is less well characterized as there has been no dose-volume correlate that is predictive of late cardiac events. This is likely due to shorter follow up data on SBRT patients and treatment of much smaller tumors and smaller cardiac/pericardial volumes in the SBRT setting (27).

\section{Current practice for central tumors}

The final results of the NRG Oncology/RTOG 0813 doseescalation trial (5) were recently published. The pivotal phase II trial tested the maximally tolerated dose (MTD) of SBRT for centrally located tumors, over a 5 -fraction schedule with radiation delivered every other day (dose per fraction ranged from 10.0 Gy per day to the MTD of 12.0 Gy). The grade 3 and above dose-limiting toxicities (DLT), defined as radiation pneumonitis, fatal pulmonary hemorrhage, hypoxia, new pleural effusion and death, were found to be $7.2 \%$. The higher-dose arms (11.5 and 12.0 Gy per fractions) achieved local control rates of $89 \%$ at 2 years. However, among patients who were evaluable for DLT, zero patients developed a severe toxicity at the $10.0 \mathrm{~Gy}$ per fraction level, compared to the higher dose groups at 10.5 Gy per fraction or higher. These higher dose cohorts developed at least 1 DLT which was grade 3 or above. As a result, most radiation oncologists would recommend that the central lung tumoral dose be kept at 10.0 Gy per fraction, for 5 fractions $(50 \mathrm{~Gy}$ total) $(1,28)$. Other acceptable dose regimens include, $9.0 \mathrm{~Gy} \times 5$ fractions (45 Gy total), $7.5 \mathrm{~Gy}$ $\times 8$ fractions (60 Gy total), and $12.5 \mathrm{~Gy} \times 4$ fractions (50 Gy total) (29-33).

Several studies, including RTOG 0813 which is discussed above, have evaluated patient outcomes after SBRT for central lung tumors. The 2-year local control after central and ultracentral lung SBRT are reported to range from $79 \%$ to $90 \%$ with 2 - and 3 -year overall survival reported as $43 \%$ to $80 \%$ respectively $(5,29,31-37)$. These are excellent outcomes despite the patients' significant pre-existing pulmonary comorbidities and smoking history $(5,29,31-37)$. However, there still exists a small percentage (5\% to $7.5 \%$ or less) of possible treatment-related deaths (grade 5 toxicities) associated with SBRT for central lung tumors (5,29,31-37). Most of these series included ultracentral lesions, and two studies focused particularly on ultracentral lesions $(35,37)$. Potentially fatal side effects may include radiation-related pneumonitis, central airway necrosis (38), tracheoesophageal fistula, or bronchopulmonary hemorrhage $(5,29-33)$.

\section{Current practice for ultracentral lung tumors}

Most ultracentral lung tumors are not resectable and primary therapy usually involves radiotherapy. For unresectable locally advanced non-small cell lung cancer and limited stage small cell lung cancer, the standard of care would be definitive chemoradiation. There are practice considerations that could limit the dose to the heart, lung, and esophagus to try to mitigate acute and late toxicities from definitive chemoradiation.

For conventionally fractionated (1.8-2.0 Gy/fraction) radiotherapy, one consideration is proton delivery for bulky tumors where the mean lung dose or V20 are exceeded by an intensity modulated radiation therapy (IMRT) photon plan $(39,40)$. For tumors that do not about the esophagus, one could try to exclude dose to the esophagus to minimize mean esophageal dose thereby reducing the risk of both acute esophagitis and late stricture. Similarly, there have been multiple recently published data on limiting the mean heart dose using either an IMRT or proton technique $(20,22)$. In the end, while the radiation plan attempts to minimize toxicities, it is also important to balance the implications of recurrence with undercoverage of the target.

For small lesions (T1 and small T2) that are ultracentral, SBRT has been utilized safely and effectively (37,41-43). For patients with node negative disease but T3 and above tumors who are not candidates for chemoradiation, some investigators have treated these with hypofractionated radiotherapy in an attempt to achieve local control or SBRT type doses $(6,35,44)$. However, the standard of care for the treatment of ultracentral tumors continues to be a subject of heated debate among radiation oncologists.

\section{Controversies in central and ultracentral lung treatments}

The greatest controversy for both central and ultracentral lung radiation is the incidence of toxicity and the safest dose regimen for radiotherapy delivery. There have been multiple single institution studies examining the outcomes of SBRT delivered in 8 fractions or less to ultracentral lesions (29-33). Memorial Sloan Kettering noted that tumors that were located closest to the proximal bronchial tree had 
the highest risk of treatment-related death attributable to radiation-associated airway necrosis (18). The VU Center in Europe has also published their ultracentral SBRT series with a very high rate $(15 \%)$ of fatal pulmonary hemorrhage using 60 Gy in 12 fractions, a relatively modest hypofractionated regimen (35). Similarly, a Chinese study in which doses as low as 35 Gy in 5 fractions delivered for ultracentral lesions still showed a $3.9 \%$ incidence of treatment-related death in 51 patients and a $9.8 \%$ risk of grade 3 or higher toxicity (37).

There is limited prospective data as the original definition of central lung lesions were those within $2 \mathrm{~cm}$ of the proximal bronchial tree. As discussed above, NRG/ RTOG 0813 was a prospective central lung SBRT dose escalation trial that showed no maximum tolerated toxicity even to a dose of $60 \mathrm{~Gy} / 5$ fractions although analysis of ultracentral $v s$. central location is still pending (5). Ongoing radiation studies are attempting to examine the optimal dose schema including the Canadian SUNSET trial that starts at 60 Gy in 8 fractions (43) and the EORTC LungTech study which also employs the $60 \mathrm{~Gy}$ in 8 fraction regimen (45). There are also trials examining multimodality therapy for ultracentral lesions. One such trial was published by UCLA in which thermal ablation and SBRT were combined for lesions abutting esophagus and other critical structures (46).

\section{Future directions}

SBRT will remain an important tool for patients with central or ultracentral lung tumors. Often, it may be the only locoregional treatment option if the patients are moderately to severely compromised from a cardiopulmonary prospective. The 5 -year survival for localized non-small cell lung cancer (NSCLC) patients with untreated tumors is $7 \%$, with a 11 -month median survival (47). While the benefits of giving SBRT treatment frequently outweighs the risks in most clinical scenarios, the consistently reported grade 5 toxicities in the $2-7 \%$ range are a real concern and further investigation is needed to minimize fatalities attributable to SBRT in this setting.

Two particular areas of future research are worth noting: First, there has been an increase in the utilization of protons in lung cancer, mainly in locally advanced cases when chemoradiation is required $(40,48,49)$. Due to a physical phenomenon called the Bragg peak effect, a proton-based radiotherapy plan can deliver full radiation dose to an intrathoracic target with radiation "stopping" and dissipating entirely beyond the target's range, resulting in virtually zero radiation dose exiting the body. This often translates to lower lung and cardiac doses. As proton technology continues to advance (mainly needing to provide a higher dose rate, and also IGRT improvement such as daily cone-beam CT's), stereotactic body proton beam therapy (SBPT) will become a reality and may offer an advantage beyond what typical photon SBRT can achieve today, especially in the setting of ultracentral and central tumors.

Secondly, in the immunotherapy era, more investigators are looking at strategies in combining checkpoint inhibitors and SBRT safely, as the ablative dose effect of SBRT can also release tumoral antigens directly and augment tumor response to immunotherapy. Data from initial clinical trials combining radiotherapy and immunotherapy show a higher rate of overall response to immunotherapy compared to immunotherapy alone $(50 \%$ vs. $20 \%, \mathrm{P}<0.1)$ in patients with advanced NSCLC (50). More clinical trials are currently ongoing.

\section{Conclusions}

In the current review, we have summarized the contemporary practice and principles of using hypofractionated RT (more than 5 fractions) or SBRT for central and ultracentral thoracic tumors, and also discussed the relevant clinical pearls and controversies associated with these techniques. As more studies combining SBRT with systemic therapy are published, the use of SBRT should see a surge; ultimately improving care for our patients in the next 5 to 10 years.

\section{Acknowledgments}

Funding: None.

\section{Footnote}

Provenance and Peer Review: This article was commissioned by the Guest Editor (Chi Wan Koo) for the series "Contemporary Practice in Thoracic Neoplasm Diagnosis, Evaluation and Treatment" published in Fournal of Thoracic Disease. The article was sent for external peer review organized by the Guest Editor and the editorial office.

Conflicts of Interest: Both authors have completed the ICMJE uniform disclosure form (available at: http://dx.doi. org/10.21037/jtd-2019-cptn-01). The series "Contemporary Practice in Thoracic Neoplasm Diagnosis, Evaluation 
and Treatment" was commissioned by the editorial office without any funding or sponsorship. DO has no financial or non-financial interests to be declared. TTS reports non-financial support from Novocure, Inc., outside the submitted work.

Ethical Statement: The authors are accountable for all aspects of the work in ensuring that questions related to the accuracy or integrity of any part of the work are appropriately investigated and resolved.

Open Access Statement: This is an Open Access article distributed in accordance with the Creative Commons Attribution-NonCommercial-NoDerivs 4.0 International License (CC BY-NC-ND 4.0), which permits the noncommercial replication and distribution of the article with the strict proviso that no changes or edits are made and the original work is properly cited (including links to both the formal publication through the relevant DOI and the license). See: https://creativecommons.org/licenses/by-nc-nd/4.0/.

\section{References}

1. Sio TT, Mohindra P, Yu NY, et al. The Search for Optimal Stereotactic Body Radiotherapy Dose in Inoperable, Centrally Located Non-Small-Cell Lung Cancer Continues. J Clin Oncol 2019;37:2697-9.

2. Nagata $Y$, Kimura T. Stereotactic body radiotherapy (SBRT) for Stage I lung cancer. Jpn J Clin Oncol 2018;48:405-9.

3. Dieterich S, Green O, Booth J. SBRT targets that move with respiration. Phys Med 2018;56:19-24.

4. Timmerman R, McGarry R, Yiannoutsos C, et al. Excessive toxicity when treating central tumors in a phase II study of stereotactic body radiation therapy for medically inoperable early-stage lung cancer. J Clin Oncol 2006;24:4833-9.

5. Bezjak A, Paulus R, Gaspar LE, et al. Safety and Efficacy of a Five-Fraction Stereotactic Body Radiotherapy Schedule for Centrally Located Non-Small-Cell Lung Cancer: NRG Oncology/RTOG 0813 Trial. J Clin Oncol 2019;37:1316-25.

6. Palma DA. An Ultracentral Lung Tumor. Int J Radiat Oncol Biol Phys 2017;97:651.

7. Stauder MC, Macdonald OK, Olivier KR, et al. Early pulmonary toxicity following lung stereotactic body radiation therapy delivered in consecutive daily fractions. Radiother Oncol 2011;99:166-71.
8. Abdel-Rahman O. Validation of the AJCC 8th lung cancer staging system among patients with small cell lung cancer. Clin Transl Oncol 2018;20:550-6.

9. Yang L, Wang S, Zhou Y, et al. Evaluation of the 7(th) and 8(th) editions of the AJCC/UICC TNM staging systems for lung cancer in a large North American cohort. Oncotarget 2017;8:66784-95.

10. Akthar AS, Ferguson MK, Koshy M, et al. Limitations of PET/CT in the Detection of Occult N1 Metastasis in Clinical Stage I(T1-2aN0) Non-Small Cell Lung Cancer for Staging Prior to Stereotactic Body Radiotherapy. Technol Cancer Res Treat 2017;16:15-21.

11. Decaluwe H, Stanzi A, Dooms C, et al. Central tumour location should be considered when comparing $\mathrm{N} 1$ upstaging between thoracoscopic and open surgery for clinical stage I non-small-cell lung cancer. Eur J Cardiothorac Surg 2016;50:110-7.

12. Decaluwe H, De Ruysscher D, D'Amico T. Location of the Tumor is a "Central" Predictor of Nodal (N1) Upstaging. J Thorac Oncol 2016;11:e89-90.

13. Ettinger DS, Wood DE, Aggarwal C, et al. NCCN Guidelines Insights: Non-Small Cell Lung Cancer, Version 1.2020. J Natl Compr Canc Netw 2019;17:1464-72.

14. Kalemkerian GP, Loo BW, Akerley W, et al. NCCN Guidelines Insights: Small Cell Lung Cancer, Version 2.2018. J Natl Compr Canc Netw 2018;16:1171-82.

15. Horne ZD, Richman AH, Dohopolski MJ, et al. Stereotactic body radiation therapy for isolated hilar and mediastinal non-small cell lung cancers. Lung Cancer 2018;115:1-4.

16. Oskan F, Becker G, Bleif M. Specific toxicity after stereotactic body radiation therapy to the central chest : A comprehensive review. Strahlenther Onkol 2017;193:173-84.

17. Yoo GS, Oh D, Pyo H, et al. Concurrent chemoradiotherapy for unresectable non-small cell lung cancer invading adjacent great vessels on radiologic findings: is it safe? J Radiat Res 2019;60:234-41.

18. Haseltine JM, Rimner A, Gelblum DY, et al. Fatal complications after stereotactic body radiation therapy for central lung tumors abutting the proximal bronchial tree. Pract Radiat Oncol 2016;6:e27-33.

19. Dess RT, Sun Y, Muenz DG, et al. Cardiac Dose in Locally Advanced Lung Cancer: Results From a Statewide Consortium. Pract Radiat Oncol 2020;10:e27-e36.

20. Dess RT, Sun Y, Matuszak MM, et al. Cardiac Events After Radiation Therapy: Combined Analysis of Prospective 
Multicenter Trials for Locally Advanced Non-Small-Cell

Lung Cancer. J Clin Oncol 2017;35:1395-402.

21. Yegya-Raman N, Wang K, Kim S, et al. Dosimetric Predictors of Symptomatic Cardiac Events After Conventional-Dose Chemoradiation Therapy for Inoperable NSCLC. J Thorac Oncol 2018;13:1508-18.

22. Wang K, Pearlstein KA, Patchett ND, et al. Heart dosimetric analysis of three types of cardiac toxicity in patients treated on dose-escalation trials for Stage III non-small-cell lung cancer. Radiother Oncol 2017;125:293-300.

23. Wang K, Eblan MJ, Deal AM, et al. Cardiac Toxicity After Radiotherapy for Stage III Non-Small-Cell Lung Cancer: Pooled Analysis of Dose-Escalation Trials Delivering 70 to 90 Gy. J Clin Oncol 2017;35:1387-94.

24. McWilliam A, Kennedy J, Hodgson C, et al. Radiation dose to heart base linked with poorer survival in lung cancer patients. Eur J Cancer 2017;85:106-13.

25. Bradley JD, Hu C, Komaki RR, et al. Long-Term Results of NRG Oncology RTOG 0617: Standard- Versus HighDose Chemoradiotherapy With or Without Cetuximab for Unresectable Stage III Non-Small-Cell Lung Cancer. J Clin Oncol 2020;38:706-14.

26. Chun SG, Hu C, Choy H, et al. Impact of IntensityModulated Radiation Therapy Technique for Locally Advanced Non-Small-Cell Lung Cancer: A Secondary Analysis of the NRG Oncology RTOG 0617 Randomized Clinical Trial. J Clin Oncol 2017;35:56-62.

27. Reshko LB, Kalman NS, Hugo GD, et al. Cardiac radiation dose distribution, cardiac events and mortality in early-stage lung cancer treated with stereotactic body radiation therapy (SBRT). J Thorac Dis 2018;10:2346-56.

28. Bezjak A, Paulus R, Gaspar LE, et al. Reply to T. Sio et al. J Clin Oncol 2019;37:2699-700.

29. Arnett ALH, Mou B, Owen D, et al. Long-term Clinical Outcomes and Safety Profile of SBRT for Centrally Located NSCLC. Adv Radiat Oncol 2019;4:422-8.

30. Chang JY, Li QQ, Xu QY, et al. Stereotactic ablative radiation therapy for centrally located early stage or isolated parenchymal recurrences of non-small cell lung cancer: how to fly in a "no fly zone". Int J Radiat Oncol Biol Phys 2014;88:1120-8.

31. Modh A, Rimner A, Williams E, et al. Local control and toxicity in a large cohort of central lung tumors treated with stereotactic body radiation therapy. Int J Radiat Oncol Biol Phys 2014;90:1168-76.

32. Roach MC, Robinson CG, DeWees TA, et al. Stereotactic Body Radiation Therapy for Central Early-Stage NSCLC:
Results of a Prospective Phase I/II Trial. J Thorac Oncol 2018;13:1727-32.

33. Tekatli H, Senan S, Dahele M, et al. Stereotactic ablative radiotherapy (SABR) for central lung tumors: Plan quality and long-term clinical outcomes. Radiother Oncol 2015;117:64-70.

34. Chang JH, Poon I, Erler D, et al. The safety and effectiveness of stereotactic body radiotherapy for central versus ultracentral lung tumors. Radiother Oncol 2018;129:277-83.

35. Tekatli H, Haasbeek N, Dahele M, et al. Outcomes of Hypofractionated High-Dose Radiotherapy in Poor-Risk Patients with "Ultracentral" Non-Small Cell Lung Cancer. J Thorac Oncol 2016;11:1081-9.

36. Cong Y, Xuan L, Sun B, et al. Retrospective comparison of stereotactic body radiotherapy versus intensity-modulated radiotherapy for stage III ultra-central squamous nonsmall-cell lung cancer. Future Oncol 2019;15:1855-62.

37. Cong Y, Sun B, Wang J, et al. Outcomes and toxicity of stereotactic body radiation therapy for advanced stage ultra-central non-small cell lung cancer. Thorac Cancer 2019;10:1567-75.

38. Corradetti MN, Haas AR, Rengan R. Central-airway necrosis after stereotactic body-radiation therapy. N Engl J Med 2012;366:2327-9.

39. Mesko S, Gomez D. Proton Therapy in Non-small Cell Lung Cancer. Curr Treat Options Oncol 2018;19:76.

40. Yu NY, DeWees TA, Liu C, et al. Early Outcomes of Patients With Locally Advanced Non-small Cell Lung Cancer Treated With Intensity-Modulated Proton Therapy Versus Intensity-Modulated Radiation Therapy: The Mayo Clinic Experience. Adv Radiat Oncol 2019;5:450-8.

41. Yang D, Cui J, Zhao J, et al. Stereotactic ablative radiotherapy of $60 \mathrm{~Gy}$ in eight fractions is safe for ultracentral non-small cell lung cancer. Thorac Cancer 2020;11:754-61.

42. Nguyen KNB, Hause DJ, Novak J, et al. Tumor Control and Toxicity after SBRT for Ultracentral, Central, and Paramediastinal Lung Tumors. Pract Radiat Oncol 2019;9:e196-e202.

43. Giuliani M, Mathew AS, Bahig H, et al. SUNSET: Stereotactic Radiation for Ultracentral Non-Small-Cell Lung Cancer-A Safety and Efficacy Trial. Clin Lung Cancer 2018;19:e529-e32.

44. Verma V, Shostrom VK, Zhen W, et al. Influence of Fractionation Scheme and Tumor Location on Toxicities After Stereotactic Body Radiation Therapy for Large ( $>/=5$ 
cm) Non-Small Cell Lung Cancer: A Multi-institutional Analysis. Int J Radiat Oncol Biol Phys 2017;97:778-85.

45. Adebahr S, Collette S, Shash E, et al. LungTech, an EORTC Phase II trial of stereotactic body radiotherapy for centrally located lung tumours: a clinical perspective. Br J Radiol 2015;88:20150036.

46. Sandler KA, Abtin F, Suh R, et al. A Prospective Phase 2 Study Evaluating Safety and Efficacy of Combining Stereotactic Body Radiation Therapy With Heat-based Ablation for Centrally Located Lung Tumors. Int J Radiat Oncol Biol Phys 2018;101:564-73.

47. Jeppesen SS, Hansen NCG, Schytte T, et al. Survival of localized NSCLC patients without active treatment or treated with SBRT. Acta Oncol 2018;57:219-25.

48. Kadoya N, Obata Y, Kato T, et al. Dose-volume

Cite this article as: Owen D, Sio TT. Stereotactic body radiotherapy (SBRT) for central and ultracentral node-negative lung tumors. J Thorac Dis 2020;12(11):7024-7031. doi: 10.21037/ jtd-2019-cptn-01 comparison of proton radiotherapy and stereotactic body radiotherapy for non-small-cell lung cancer. Int J Radiat Oncol Biol Phys 2011;79:1225-31.

49. Nantavithya C, Gomez DR, Wei X, et al. Phase 2 Study of Stereotactic Body Radiation Therapy and Stereotactic Body Proton Therapy for High-Risk, Medically Inoperable, Early-Stage Non-Small Cell Lung Cancer. Int J Radiat Oncol Biol Phys 2018;101:558-63.

50. Theelen WSME, Peulen HMU, Lalezari F, et al. Effect of Pembrolizumab After Stereotactic Body Radiotherapy vs Pembrolizumab Alone on Tumor Response in Patients With Advanced Non-Small Cell Lung Cancer: Results of the PEMBRO-RT Phase 2 Randomized Clinical Trial. JAMA Oncol 2019;5:1276-82. 